\title{
Barking up the right tree
}

DOI:

10.1038/nrc2017

\section{URLs \\ VEGF}

http://www.ncbi.nlm.nih.gov/

entrez/query.fcgi?db=gene\&c

$\mathrm{md}=$ Retrieve\&dopt=full_

report\&list_uids $=7422$

VEGFR2

http://www.ncbi.nlm.nih.gov/ entrez/query.fcgi?db=gene\&c $\mathrm{md}=$ Retrieve\&dopt=full_ report\&list_uids=3791

Pancreatic cancer http://www.cancer.gov/ cancertopics/types/pancreatic

Lung cancer http://www.cancer.gov/ cancertopics/types/lung
The treatment of solid tumours with a vascular endothelial growth factor (VEGF) inhibitor can lead to the loss of most of the tumour vasculature. But just how much of the vasculature actually dies? Donald McDonald and colleagues have found that, like a deciduous tree in winter, structural components of the vascular tree remain and can aid the rapid growth of new vessels once the inhibitor is removed.

When mice with tumours are treated with VEGF inhibitors, dramatic changes in the tumour vasculature are seen, including the cessation of new vessel formation, the loss of most tumour vessels and the normalization of any that remain. But tumour vessels grow back rapidly once treatment is stopped; how is this achieved? It is the endothelial cells (the cells that line the vessels) that die when treated with VEGF inhibitors, the vascular basement membrane and many of the pericytes (vascular-support cells) remain. McDonald and colleagues asked if these remnants assist vascular re-growth.

Re-growth of the tumour vessels was analysed after 7 days of treatment with the VEGF receptor (VEGFR) tyrosine kinase inhibitor AG-013736 in mice with either transgene-induced pancreatic $\beta$-cell tumours or in mice implanted with Lewis lung carcinomas. Once the inhibitor was withdrawn, the tumour vasculature re-grew rapidly, and had completely re-grown in both tumour types 7 days after anti-VEGF treatment had ended. Importantly, re-growth was accompanied by an increase in the expression of VEGFR2 in endothelial cells, indicating that the re-growing vessels were VEGF dependent. This was verified by treating again with AG-013736, which led to the regression of these vessels.

The authors noted that as the endothelial cells died, the pericytes and the basement membrane tracts, in the form of empty sleeves of type IV collagen, remained. Moreover, neither pericyte cell number nor levels of type IV collagen increased once the vessels re-grew, indicating that the tracts of basement membrane might be re-used by the re-growing vessels. Indeed, as the vessels re-grew, the empty basement membrane tracts were lost, and vascular endothelial sprouts could be seen in these tracts during the initial stages of re-growth. The authors also found that VEGF, which is known to bind basement-membrane components, accumulated in the empty tracts and probably provided a directional stimulus for the re-growing vessels.

The authors conclude that destroying pericytes and the vascular basement membrane, which functions as a scaffold for the re-growing endothelial cells, might complement anti-VEGF therapies by further decreasing the potential for vessel re-growth.

Nicola McCarthy

ORIGINAL RESEARCH PAPER Mancuso, M. R. et al. Rapid vascular regrowth in tumours after reversal of VEGF inhibition. J. Clin. Invest. 116, 2610-2621 (2006)

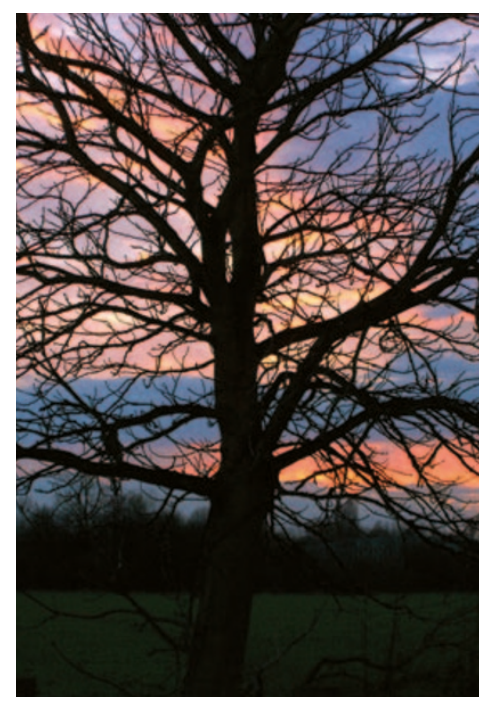

